\title{
SÍNDROME DE KOCHER-DEBRÉ-SEMELAIGNE
}

\author{
J. ForTes-Rego *
}

A deficiência congênita da glândula tireóide determina um defeito no desenvolvimento corporal e cretinismo ${ }^{9}$. Em 1892, Kocher, citado por Norris e Panner ${ }^{22}$, descreveu, pela primeira vez, a ocorrência de alterações musculares (aumento das massas musculares, debilidade e lentidão nos movimentos) em pacientes com cretinismo ${ }^{22}$. Em 1934, Debré e Semelaigne ${ }^{6}$ comunicaram à Sociedade de Pediatria de Paris a mesma associação, sob o título de "hipertrofia muscular generalizada da criança" e, no ano subseqüente, deram a conhecer, a história completa de dois casos ${ }^{7}$. A partir de então foi-lhe atribuída a designação de síndrome de Kocher-Debré-Semelaigne, embora na literatura francesa, onde se registra a maior parte dos casos, apareça quase sempre sem alusão ao autor suiço, negando-se-lhe sua prioridade cronológica.

Vale a pena ressaltar que o termo hipertrofia é usado aqui no sentido estritamente morfológico, sem implicações na estrutura histológica do músculo ${ }^{5,21,26}$, havendo mesmo autores, a exemplo de Giovannucci ${ }^{13}$, que falam de pseudohipertrofia, enquanto outros, como Indira Bai e col. ${ }^{16}$, referem-se a hiperplasia muscular com tendência à atrofia. Spiro e col. ${ }^{29}$, em estudos histoquímico e ultraestrutural realizados em biopsia muscular obtida de uma criança com síndrome de Kocher-Debré-Semelaigne, enumeram várias alterações qualificadas de inespecificas, as quais têm sido encontradas também em hipotiroidismo induzido em animais; tal conceito contraria alguns estudos anteriores sobre eventual especificidade das lesões musculares no hipotiroidismo ${ }^{10,18}$. A hipertrofia é generalizada, envolvendo particularmente os músculos das extremidades, dando à criança uma aparência "atlética", quase "hercúlea". Os transtornos metabólicos em jogo são desconhecidos ${ }^{19}$.

Uma outra miopatia hipotiroidiana que deve ser referida graças à afinidade que apresenta com o quadro que ora nos ocupa é a síndrome de Hoffmann, individualizada pela presença de miotonia e aparecimento no adulto. Alguns autores, porém, estribados no relato de casos de sindrome de Kocher-Debré-Semelaigne em adultos e na ocorrência da síndrome de Hoffmann em crianças, consideram-nas como variantes de um mesmo processo mórbido. Moris e col. ${ }^{22}$, partidários desta opinião, reportam um caso de síndrome de Kocher-Debré-Semelaigne em adulto que, durante tratamento

Trabalho do Departamento de Medicina Especializada da Faculdade de Ciências da Saúde da Universidade de Brasilia, apresentado ao VI Congresso da Academia Brasileira de Neurologia (Rio de Janeiro, julho de 1974): * Professor Assistente. 
com tiroxina, "evoluiu" para uma síndrome de Hoffmann, com o surgimento de miotonia.

Formas ainda mais raras de acometimento neuromuscular na disfunção tiroidiana têm sido descritas ${ }^{1,2,14,15,23,27,30 .}$

\section{OBSERVAÇÃO}

N. N. C., com 8 anos de idade, sexo masculino, moreno, atendido pela primeira vez em 25-08-1972 em consulta externa no ambulatório médico da Escola Especial "Ana Cordeiro" (Teresina), onde fora matriculado aos 5 anos, na condiçáo de "excepcional". A genitora referia que desde o nascimento notara ser a crianca doente, tendo passado os 6 primeiros dias de vida praticamente sem receber alimentos, "vivendo unicamente de remédios". Antecedentes familiares - Pai com 46 anos, aparentemente sadio e sem vícios. Mãe com 40 anos, obesa, considerando-se "muito doente dos nervos". Doze irmãos vivos, sendo que um, atualmente com 16 anos, padece hipoacusia bilateral desde tenra idade. Antecedentes pessoais - Gestação a termo, parto normal porém prolongado, evidenciando intensa cianose neonatal; pesou, ao nascer, 6.200 gramas (sic). Tardou em caminhar e falar (diz apenas "mamá" e "papá"). Em 1971 fora operado de hérnia. Exame neurológico - A inspeção, chama a atenção uma estatura demasiado pequena para a idade $(83 \mathrm{~cm})$, contrastando com um perimetro cefálico avantajado $(53 \mathrm{~cm})$; cabelo escasso e grosso; nariz chato, hipertelorismo; boca permanentemente entreaberta; abdomen saliente; musculatura esquelética universalmente hipertrofiada; pele grossa e seca (Fig. 1). Acentuada inércia; não responde, nem cumpre ordens. Objetivamente, não há sinais de lateralização. Exames complementares - Hemograma: discreta anemia. Urina normal. Testes sangüineos para lues negativos. Colesterolemia $357 \mathrm{mg} \%$. Radiografias de ossos longos, mãos e pés: retardo de ossificação (Fig. 2). Radiografia do tórax: aumento da área cardíaca. ECG de baixa voltagem. EEG normal. Biopsia muscular: "Observa-se tecido muscular estriado com fibras de tamanho variável, encontrando-se algumas atróficas, sem a estriação transversal característica. Ocorre fragmentação focal e se esboça a proliferação fibroblástica" (Dr. José Figueiredo da Silva - Hospital Getúlio Vargas, Teresina). Tratamento - Tireóide dessecada em doses progressivas até atingir $25 \mathrm{mg} / \mathrm{dia}$, de forma contínua. Não houve manifestações de intolerância.

Evolução - As primeiras modificaçōes relatadas pelos familiares, após um mês de iniciado o tratamento, consistiram numa redução no grau de inércia e na proemiência abdominal. No último contato que mantivemos com o paciente, em 26-12-1973, após 15 meses de tratamento, com pequenas interrupçōes, pudemos constatar total regressão da saliência abdominal e do aumento das massas musculares, importante progresso na atividade motora, maior interesse pelos entretenimentos, sobretudo em grupo e um enriquecimento do vocabulário, com adquisição de algumas novas palavras, além de um crescimento de $16 \mathrm{~cm}$ com relação à altura consignada na consulta inicial.

\section{COMENTARIOS}

A raridade da síndrome em pauta parece-nos, por si só, justificar a presente comunicação. Não encontramos relatos similares na literatura nacional a nosso alcance. Contudo, nosso escopo precípuo, é destacar a evolução do caso, bastante favorável, sobretudo se considerarmos o grande atraso no inicio do tratamento específico, o que somente ocorrera aos 2 anos de idade. O fato contraria, assim, a opinião de muitos de que uma terapêtica 


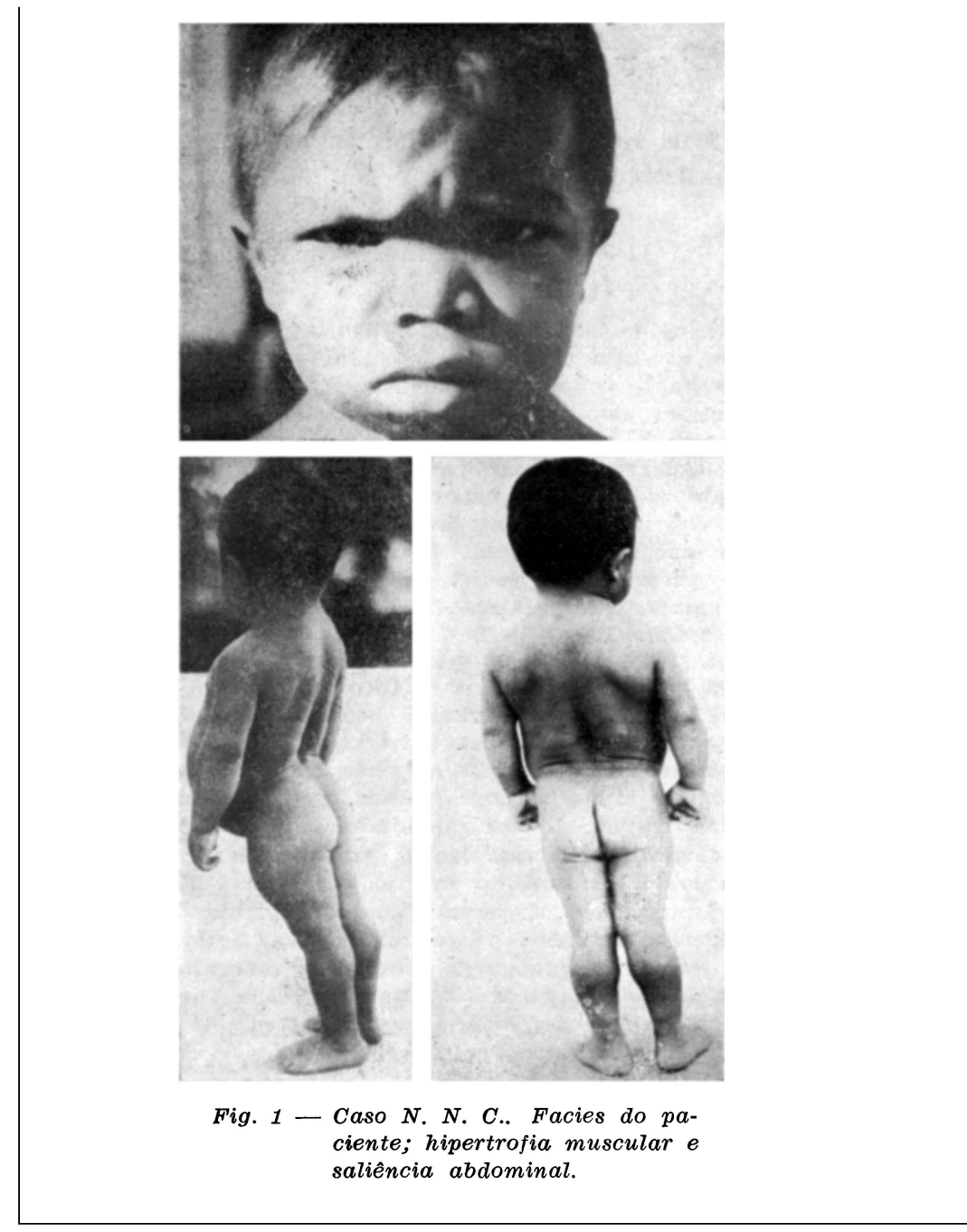

reparadora tardia não induz efeitos benéficos para este tipo de enfermidade, mormente no que diz respeito ao sistema nervoso. A substância usada foi o hormônio natural, não obstante a preferência atual da maioria dos autores pelas preparações sintéticas ${ }^{3,4,11,12,20,24,25}$. Ingbar e Woeber ${ }^{17}$ justificam-na em virtude de ser a levotiroxina capaz de inverter todas as anormalidades conhecidas do estado hipotiroidiano do adulto e pela uniformidade de sua potência. Com relação à dose, seguimos o conceito clássico de ser a mesma tão elevada quanto possível, com o limite superior determinado pelo aparecimento de eventuais efeitos colaterais. Em trabalho recente, Evered e col. ${ }^{8}$, usando a L-tiroxina, mencionam doses parcimoniosas, girando em torno de um terço das preconizadas pela maior parte dos livros textos 

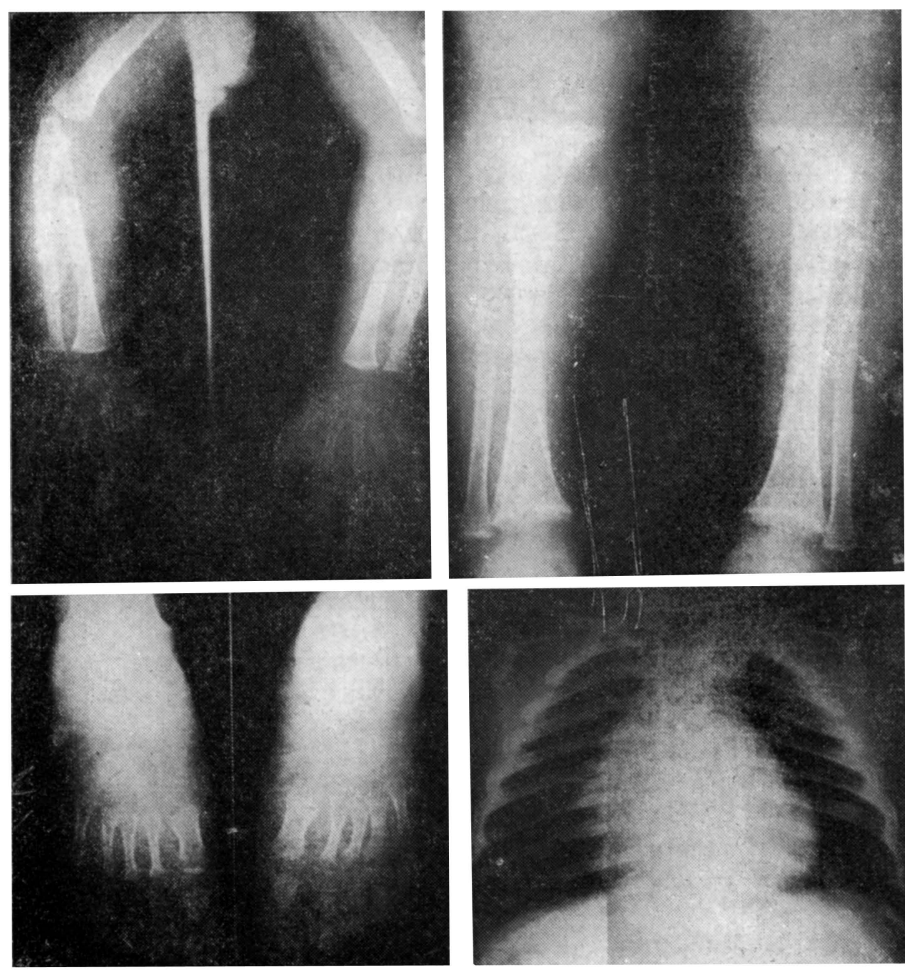

Fig. 2-Caso N. N. C.. Kudiogıalaas aos membros superiores $e$ inferiores mostrando retardo no desenvolvimento dos centros de ossificação, com separação das diáfises $e$ epifises. Radiografia de tórax mostrando aumento da área cardiaca.

de Clínica e Endocrinologia, as quais são por eles consideradas como excessivas.

\section{RESUMO}

E relatado um caso de síndrome de Kocher-Debré-Semelaigne diagnosticado em um paciente de 8 anos, não tendo sido encontrados relatos similares na literatura nacional consultada. Apesar do início tardio do tratamento, a evolução mostrou-se bastante favorável.

\section{SUMMARY}

Kocher-Debré-Semelaigne syndrome: a case report

A case of Kocher-Debré-Semelaigne syndrome is reported and a critical review of the literature is made. The patient responded well to extract 
thyroid with improvement of hypothyroidism and diminution in the size of the muscles.

\section{REFERENCIAS}

1. ASTROM, K. E.; KUGELBERG, E. \& MULLER, R. - Hypothyroid myopathy. Arch. Neurol. (Chicago) 5:472, 1961.

2. BERGOUIGNAN, M.; VITAL, CL. \& BATAILLE, J. M. - Les myopathies hypothyroidiènnes. Aspects cliniques et histopathologiques. Prèsse Méd. 75:1551, 1967.

3. BRAVERMAN, L. E.; INGBAR, S. H. \& STERLING, K. - Conversion of thyroxine (T4) to trilodothyronine (T3) in athyreotic human subjects. J. Clin. Invest. 49:855, 1970.

4. COTTON, G. E.; GORMAN, C. A. \& MAYBERRY, W. E. - Supression of thyrotropin ( $h$-TSH) in serums of patients with myxedema of varying etiology treated with thyroid hormones. New England J. Med. 285:529, 1971.

5. DARRE, H.; MOLLARET, P.; ZAGDOUN, M. \& OEHMICHEN, M. - Hypertrophie musculaire généralisée du nourrison et hypothyroidie congénitale. Rev. Neurol. (Paris) 72:249, 1939.

6. DEBRE, R. \& SEMELAIGNE, G. - Hyperthrophie musculaire généralisée du petit enfant. Bull. Soc. Péd. de Paris 33:699, 1934.

7. DEBRE, R. \& SEMELAIGNE, G. - Syndrome of diffuse muscular hypertrophy in infants causing athletic appearance. Amer. J. Dis. Child. 50:1351, 1935.

8. EVERED, D.; YOUNG, E. T.; ORMSTON, B. J.; MENZIES, R.; SMITH, P. A. \& HALL, $\mathrm{R}$ - Treatment of hypothyroidism: a reappraisal of thyroxine therapy. Brit. Med. J. 2:131, 1973.

9. FORD, F. R. - Enfermedades del Sistema Nervioso en la Infancia, Niñez y Adolescencia. 2a Ed. castelhana. Editorial La Médica, Cordoba, Argentina, 1967.

10. FOSTER, M. \& BARR, D. - Myxedema: record of an autopsied case with special emphasis upon lesion of muscles. J. Clin. Endocrin. 4:417, 1944.

11. FOWLER, P. B. S. - Treatment of hypothyroidism. Brit. Med. J. 2:352, 1973.

12. FOWLER, P. B. S.; SWALE, J. \& ANDREWS, H. - Hypercholesterolemia in borderline hypothyroidism. Lancet II:488, 1970.

13. GIOVANNUCCI, M. L. - La sindrome di Kocher-Debré-Semelaigne. Min. Pediat. 22:1101, 1970.

14. HALL, B. E.; SUNDERMAN, F. W. \& GITTINGS, J. C. - Congenital muscular hypertrophy. Amer. J. Dis. Child. 52:773, 1936.

15. HEUYER, G.; LEBOUICI, S.; KOUPERNIK, C. \& MARTINETTI, J. - Myxoedème congénital, hypertrophie musculaìre généralisée, malformation vertébrale et atrophie corticale. Arch. Franc. Pédiat. 7:968, 1950.

16. INDIRA BAI, K.; SUBHA RAO, K. V. \& VIJAYALAKSHMI, B. - The Kocher-Debré-Semelaigne syndrome. Ind. Pediat. 8:852, 1971.

17. INGBAR, S. H. \& WOEBER, K. A. - Tratado de Endocrinologia. Editado por R. H. Williams. $3^{a}$ Ed. castelhana. Salvat Editores, Barcelona, 1969.

18. KIRCHHEINER, B. - Specific muscle lesions in pituitary-thyroid disorders. Acta Med. Scandinavica 172:539, 1962.

19. KOBIELOWA, Z; NIWELINSKI, S.; KRZANOWSKA-DYRAS, M. \& MAZUREK, A. - Muscle hypertrophy in hypothyroidism (Debré-Semelaigne syndrome). Polish Endocrin. 21:388, 1970.

20. LARSEN, P. R. - Triiodothyronine: review of recent studies of its physiology and pathophysiology in man. Metab. Clin. Experim. 21:1073, 1972.

21. NAJJAR, S. S. \& NACHMAN, H. S. - The Kocher-Debré-Semelaigne syndrome. J. Pediat. 66:901, 1965.

22. NORRIS, F. H. \& PANNER, B. J. - Hypothyroid myopathy. Arch. Neurol. (Chicago) 14:574, 1966. 
23. NORRIS, S. H.; PANNER, B. J. \& STORMONT, J. H. - Thyrotoxic periodic paralysis. Arch. Neurol. (Chicago) 19:88, 1968.

24. ORMSTON, B. J.; GARRY, R.; CRYER, R. J.; BESSER, G. M. \& HALL, R. - Thyrotrophin-releasing hormone as a thyroid-function test. Lancet II:10, 1971.

25. PITTMAN, C. S.; CHAMBERS, J. B. \& READ, V. H. - The extrathyroidal conversion rate of thyroxine to triodothyronine in normal man. J. Clin. Investig. 50:1187, 1971.

26. PUROHIT, K. R. \& MURTHY, D. - The Kocher-Debré-Semelaigne syndrome: hypothyroidism with muscular "hypertrophy". Ind. Pediat. 6:338, 1969.

27. RIMBAUD, L. \& PASSOUANT, P. - Les dystrophies musculaires des hypothyroidiens. Rev. Neurol. (Paris) 79:81, 1947.

28. SCHWARZ, G. A. \& ROSE, E. - Neuromyopathies and thyroid dysfunction. Arch. Internal Med. 112:555, 1963.

29. SPIRO, A. J.; HIRANO, A.; BEILIN, R. L. \& FINKELSTEIN, J. W. - Cretinism with muscular hypertrophy (Kocher-Debre-Semelaigne syndrome). Arch. Neurol. (Chicago) 23:340, 1970.

30. WILSON, J. \& WALTON, J. N. - Some muscular manifestitions of hypothyroidism. J. Neurol. Neurosurg. Psychiat. 22:320, 1959.

Universidade de Brasilia - Unidade Integrada de Saúde de Sobradinho 70400 Sobradinho $D F-$ Brasil. 